\title{
Notas nomenclaturais em Begonia L. (Begoniaceae) ${ }^{1}$
}

\author{
ELIANE L. JACQUES ${ }^{2,4}$ e MARIA CANDIDA H. MAMEDE ${ }^{3}$
}

(recebido: 11 de novembro de 2004; aceito: 22 de junho de 2005)

\begin{abstract}
Nomenclatural notes in Begonia L. (Begoniaceae)). As a result of a taxonomic study on the genus Begonia, 31 new synonyms are proposed, 13 lectotypes are designated and eight taxa are considered of uncertain identity. Eight invalid names are listed under Begonia fischeri Schrank, and the correct citation of the type material of Begonia raulinii Brade is presented. The rehabilitation of Begonia hammoniae Irmsch. is proposed, and nine taxa are excluded from the synonymy of various species as they are classified as cultivars or hybrids.
\end{abstract}

Key words - Begonia, Begoniaceae, nomenclature

RESUMO - (Notas nomenclaturais em Begonia L. (Begoniaceae)). Como parte dos estudos taxonômicos no gênero Begonia, são propostos neste trabalho 31 sinônimos novos, designados 13 lectótipos e oito táxons considerados de identidade duvidosa. Oito nomes não validamente publicados foram incluídos na sinonímia de Begonia fischeri Schrank, e procedeu-se à correção da citação de um material-tipo (B. raulinii Brade). Begonia hammoniae Irmsch. é reabilitada, enquanto nove táxons foram excluídos da sinonímia de várias espécies, por terem sido considerados cultivares ou híbridos.

Palavras-chave - Begonia, Begoniaceae, nomenclatura

\section{Introdução}

Begonia L. é um gênero amplamente distribuído nos trópicos e subtrópicos do Velho e Novo Mundos, com cerca de 1.400 espécies (De Lange \& Bouman 1999), classificadas em 78 seções (Baranov \& Barkley 1974). Nas Américas ocorrem cerca de 530 espécies, na África (incluindo Madagascar) cerca de 150 espécies e, na Ásia, cerca de 520 espécies (Doorenbos et al. 1998). No Brasil, está representado por cerca de 200 espécies encontradas em todas as formações vegetais, exceto nos manguezais. O gênero apresenta ampla variabilidade morfológica, podendo ser caracterizado pela assimetria da lâmina foliar, pelas flores estaminadas com quatro tépalas e pistiladas com cinco, ovário ínfero com placentação axilar e cápsulas trialadas. Durante a realização de um estudo revisional de um grupo de espécies brasileiras (Jacques 2002), além da descoberta de táxons novos (Jacques \& Mamede 2004), foram propostos vários sinônimos, encontrados nomes não validamente publicados e citações incorretas de material-tipo e selecionados

\footnotetext{
1. Parte integrante da Tese de Doutorado de E.L. Jacques, do Programa de Pós-Graduação em Botânica da Universidade de São Paulo.

2. Universidade Federal de Mato Grosso do Sul, Campus de Três Lagoas, Caixa Postal 210, 79603-011 Três Lagoas, MS, Brasil.

3. Instituto de Botânica, Caixa Postal 4005, 01061-970 São Paulo, SP, Brasil.

4. Autor para correspondência: ejacques@ceul.ufms.br
}

vários lectótipos. O presente trabalho tem por objetivo validar estas proposições.

\section{Material e métodos}

Os dados apresentados neste trabalho basearam-se em revisão bibliográfica, no estudo das populações no campo e no exame dos materiais botânicos dos seguintes herbários: ALCB, ASE, B, BAH, BHCB, BM, CEN, CEPEC, CEUL, CPAP, COR, CVRD, E, EAC, ESA, ESAL, FLOR, G, GUA, HAS, HASU, HB, HBR, HEPH, HRB, HRCB, HUEFS, IAC, IAN, IBGE, ICN, INPA, IPA, JPB, K, M, MAC, MBM, MBML, MG, P, PACA, PEUFR, PKDC, R, RB, RBR, RFA, RUSU, S, SP, SPF, UB, UEC, UFG, UFMT, UFP, UPCB, VIC (siglas de acordo com http:// sciweb.nybg.org/science2/IndexHerbariorum-asp, acessado em abril/2005).

Para confirmação das efetivas datas de publicação de algumas obras para efeito de prioridade de binômios foram consultados Carauta (1973) para os volumes da Flora Fluminensis e Stafleu \& Cowan (1979) para as demais obras.

\section{Resultados}

1. Begonia aconitifolia A. DC., Ann. Sci. Nat., Sér. 4, Bot. 11: 127. 1859. Tipo: [BRASIL]. Rio DE JANEIRo: Riedel s.n. (Holótipo LE, n.v.; Isótipo G-DC). = Begonia sceptrum Rodigas, Rev. Hort. Belge Étrangère 10(11): Tab. 253. 1884. Lectótipo, aqui designado: Rodigas, Rev. Hort. Belge Étrangère 10(11): Tab. 253. 1884, ilustração baseada em planta procedente do Brasil, "cult. J. Bot. de Bruxelas", ex descr. et icon. = Begonia faureana Linden ex Garnier, Ill. Hort. Sér. 
6, 2(10): 152, fig. 34. 1895. Lectótipo, aqui designado: Garnier, Ill. Hort. Sér. 6, 2(10): 152, fig. 34. 1895, ilustração baseada em planta procedente do Brasil, "cult.", ex descr. et icon.

= Begonia faureana Linden ex Garnier var. argentea Linden, Catalogue des orchidées et autres plantes nouvelles: Tab. 7. 1896. Lectótipo, aqui designado: Linden, Catalogue des orchidées et autres plantes nouvelles: Tab. 7. 1896, ilustração baseada em planta procedente do Brasil, "cult. J. Bot. de Bruxelas", ex descr. et icon.

Todos os sinônimos associados a B. aconitifolia foram descritos e ilustrados a partir de material cultivado e nos herbários europeus consultados não foram localizadas exsicatas que correspondessem à diagnose dos mesmos. Uma vez que nos protólogos não há indicação de material tipo, optamos pela lectotipificação das pranchas publicadas.

2. Begonia alchemilloides Meisn. ex A. DC., Ann. Sci. Nat., Sér. 4, Bot. 11: 125. 1859. Tipo: [BRASIL]. MinAS GERAIS: "in camporum editioribus runcarionibus, ad Sara, Serro Frio", V, Martius s.n. (Holótipo M). = Begonia leptophylla Taub., Bot. Jahrb. Syst. 21: 445. 1896. Tipo: [BRASIL. GoIÁs]: "in Felsspalten auf der Serra Dourada”, I-1893, Ule 310 (Holótipo HBG), syn. nov.

De Candolle (1859), ao descrever Begonia alchemilloides, incluiu-a na seção Begonia. Posteriormente, Taubert (1896) descreveu B. leptophylla posicionando-a duvidosamente na seção Pritzelia (Klotzsch) A. DC. Begonia leptophylla difere de $B$. alchemilloides unicamente pelas placentas inteiras; no entanto esta característica é bastante variável nesta última, podendo a placenta ser inteira ou bipartida em um, dois ou nos três lóculos. Além disso, nas cápsulas maduras de $B$. alchemilloides, a placenta apresenta lamelas de tamanhos extremamente diferenciados, tanto no comprimento quanto na largura, além de variações na união ou separação das lamelas. Este carácter variável não sustenta a manutenção de B. leptophylla como uma espécie distinta.

3. Begonia besleriifolia Schott in Sprengel, Syst. Veg. 4(2): 408. 1827. Tipo: BRASIL. Rio DE JANEIRO: Schott 4636 (Holótipo W, n.v.; Isótipos G, B foto).

= Begonia besleriifolia Schott var. stuhriana Brade, Arch. Jar. Bot. Rio de Janeiro 8: 235, fig. 8. 1948. Tipo: BRASIL. EsPírITo SANTo: Itaguaçu, Jatiboca, Sítio Stuhr, A.C. Brade 18589, A.B. Pereira \& A.P. Duarte (Holótipo RB), syn. nov.
Begonia besleriffolia var. stuhriana foi caracterizada pelas flores glabras (Brade 1948). A análise dos materiais-tipo desses dois táxons não justifica o reconhecimento de variedades, uma vez que ambos apresentam flores glabras.

4. Begonia fischeri Schrank, Pl. rar. hort. monac. 2(6): Tab. 59. 1819 [Maio, 1820]. Tipo: [BRASIL]. RIO DE JANEIRO: Martius s.n. (Holótipo BR ?), ex descr. et icon. = Begonia patula Fischer ex Hornem., Suppl. Hort. bot. hafn. 108. 1819, nom. illeg., non Haworth (1819). Tipo: "C. intr. 1817 ex horto Garenkano sub hoc nomine" (Holótipo C ?), ex descr.

= Begonia obliqua Vell., Fl. flumin. Icon. 10: 48. 1827 [1831]; Arq. Mus. Nac. Rio de Janeiro 5: 406. 1881, nom. illeg., non Linnaeus (1753). Lectótipo, aqui designado: Vellozo, Fl. flumin. Icon. 10: 48. 1827 [1831], ilustração baseada em planta coletada no Brasil, [Rıo de Janeiro-São Paulo], "ad declivia Alpium Fluminensium [Serra do Mar] via publica, qua e Pharmacopoli [Parati] iter fit ad oppidum Cunha".

= Begonia villosa Gardner, London J. Bot. 1: 186. 1842, nom. illeg., non Lindley (1829). Tipo: não designado (Holótipo BM ?), syn. nov., ex descr.

= Begonia palustris Hartw. ex Benth., Pl. hartw.: 184 . $1845 \equiv$ Begonia fischeri Schrank var. palustris (Benth.) Irmsch., Bot. Jahrb. Syst. 76: 24, pl. 1, fig. 7. 1953, nom. inval. $\equiv$ Begonia tovarensis Klotzsch var. palustris (Benth.) L.B. Sm. \& B.G. Schub., Caldasia 4(17): 89, fig. 10. 1946. Tipo: COLÔMBIA. "prope Popayan", 1843, Hartweg 1022 (Holótipo B; Isótipos P, E), syn. nov.

= Begonia malvacea Klotzsch, Begoniac.: 33. 1855. Begonia fischeri Schrank var. malvacea (Klotzsch) Irmsch., Bot. Jahrb. Syst. 76: 24, pl. 2, fig. 2. 1953, nom. inval. Tipo: BRASIL. Sello s.n. (Holótipo B, não localizado), syn. nov., ex descr. et icon.

= Begonia pohliana Klotzsch, Begoniac.: 33. 1855 $\equiv$ Begonia macroptera var. pohliana (Klotzsch) A. DC. in Mart., Fl. bras. 4(1): 346. 1861. Lectótipo, aqui designado: BRASIL. Pohl 1832 (B). Síntipo remanescente: BRASIL. Sello L446 (B).

= Begonia macroptera Klotzsch, Begoniac.: 34. 1855 . $\equiv$ Begonia fischeri Schrank var. macroptera (Klotzsch) Irmsch., Bot. Jahrb. Syst. 76: 24, pl. 1, fig. 5. 1953, nom. inval. Tipo: BRASIL. RIO DE JANEIRO: 1835 , Gaudichaud 1068 (Holótipo P; Isótipo B), syn. nov. = Begonia elata Klotzsch, Begoniac.: 35. 1855 $\equiv$ Begonia fischeri Schrank var. elata (Klotzsch) Irmsch., Bot. Jahrb. Syst. 76: 24, pl. 1, fig. 3. 1953, nom. inval. Lectótipo, aqui designado: BRASIL. Sello 135, 
fr. (B; Isolectótipo B, fl.), syn. nov.

= Begonia ermanii Klotzsch, Begoniac.: 35.1855

$\equiv$ Begonia uliginosa Klotzsch var. ermanii (Klotzsch) A.DC. in Mart., Fl. bras. 4(1): 347. 1861. Begonia fischeri Schrank var. ermanii (Klotzsch) Irmsch., Bot. Jahrb. Syst. 76: 24, pl. 1, fig. 6. 1953, nom. inval. Tipo: [BRASIL]. Rio DE JANEIRO: Erman s.n. (Holótipo B), syn. nov.

= Begonia moritziana Klotzsch, Begoniac.: 31. 1855 三Begonia tovarensis Moritz, nom. illeg. et pro syn., fide Klotzsch (1855) $\equiv$ Begonia fischeri Schrank var. moritziana (Klotzsch) Irmsch., Bot. Jahrb. Syst. 76: 24, pl. 2, fig. 1. 1953, nom. inval. Tipo: [VENEZUELA]. "auf der deutschen Colonie Tovar, bei Caracas", Moritz s.n. (Holótipo B, não localizado), syn. nov., ex descr. et icon.

= Begonia tovarensis Klotzsch, Begoniac.: 31. 1855 $\equiv$ Begonia fischeri Schrank var. tovarensis (Klotzsch) Irmsch., Bot. Jahrb. Syst. 76: 24, pl. 1, fig. 1. 1953, nom. inval. Tipo: "Wächst in VENEZUELA und wurde zuerst von Moschkowitz \& Siegling in Einfurt aus Samen gezogen" (Holótipo B, não localizado), syn. nov., ex descr. et icon.

= Begonia brasiliensis Klotzsch, Begoniac.: 34. 1855 三Begonia fischeri Schrank var. brasiliensis (Klotzsch) Irmsch., Bot. Jahrb. Syst. 76: 24. 1953, nom. inval. Tipo: "Brasilia meridionalis, Rio das Pedras, 1820, Sello C2191" (Holótipo B), syn. nov.

= Begonia macroptera Klotzsch var. paludum A. DC. in Mart., Fl. bras. 4(1): 346. 1861. Tipo: BRASIL. "in paludibus prope BAHIA", Salzmann 473 (Holótipo P; Isótipo G), syn. nov.

= Begonia intercedens Irmsch., Bot. Jahrb. Syst. 76(1): 97. 1953. Tipo: BRASIL. RIo GRANDE Do Sul: Ihering 399 (Holótipo SP), syn. nov.

= Begonia fischeri Schrank var. brevipilosa Irmsch., Bot. Jahrb. Syst. 76(1): 98. 1953. Tipo: BRASIL. MinAS GERAIS: Keller s.n. (Holótipo B, não localizado), syn. nov., ex descr.

$=$ Begonia fischeri Schrank var. crenatoglabra Irmsch., Bot. Jahrb. Syst. 76(1): 98. 1953. Lectótipo, aqui designado: BRASIL. São Paulo: São Paulo, Ipiranga, cult. Horto Botânico, II-1916, Luederwaldt s.n. (SP 8757). Síntipo remanescente: BRASIL. SÃo PAULO: São Paulo, Ipiranga, IV-1912, Luederwaldt s.n. (SP 8756). Síntipo excluído: BRASIL. Sello 135 (= B. elata Klotzsch), syn. nov.

= Begonia fischeri Schrank var. crenulatoglabra Irmsch., Bot. Jahrb. Syst. 76(1): 99. 1953. Tipo: BRASIL. Minas Gerais: Pouso Alegre, 2-V-1927, Hoehne s.n. (Holótipo SP 19323), syn. nov.
= Begonia fischeri Schrank var. klugii Irmsch., Bot. Jahrb. Syst. 76: 24, 99, Tab. 1, fig. 9. 1953. Tipo: PERU. San Martin: Zepelacio, próximo Moyobanba, XI-XII-1933, Klug 3389 (Holótipo US, n.v.; Isótipo B), syn. nov.

Begonia patula Fischer ex Hornem. foi introduzida em cultivo com este nome no Jardim Gorenkano em 1817. Por outro lado, B. patula Haw. já era um nome amplamente utilizado para uma planta introduzida por Anderson \& Otto no Jardim Botânico de Berlim em 1811 e mantida em cultivo no viveiro de Loddiges em 1816 (Golding 1981). Visto que B. patula Fischer ex Hornem. é um nome ilegítimo por homonimia, o primeiro nome disponível para este táxon é Begonia fischeri Schrank. Ao descrever Begonia pohliana, Klotzsch (1855) citou dois materiais, Sello s.n. e Pohl s.n., sem indicar claramente o holótipo. De Candolle (1861) considerou esta espécie como uma variedade de $B$. macroptera, e relacionou quatro materiais por ele examinados: Sello s.n. e Pohl 1832 (ambos em B), Schott 1832 e 4618 (ambos em W). No Herbário de Berlim, foram examinadas as exsicatas Sello L446 e Pohl 1832, ambas contendo etiquetas com a identificação $B$. pohliana, grafadas pelo próprio Klotzsch. Desta forma, a coleção Pohl 1832, depositada em B, foi selecionada como lectótipo de B. pohliana. Begonia fischeri var. crenatoglabra foi descrita por Irmscher (1953) com base em três coleções, como um táxon intermediário entre $B$. fischeri var. elata (margem da folha grosseiramente serreada) e $B$. fischeri var. crenulatoglabra (margem da folha finamente denteada), apresentando como característica singular, estiletes com papilas estigmáticas por todo o ramo, mesmo quando velhos. Os síntipos relacionados são Sello 135, Luederwaldt s.n. (SP 8757) e Luederwaldt s.n. (SP 8756). O material Sello 135, do herbário B, é composto de duas exsicatas (uma com flores e outra com frutos), ambas grafadas por Klotzsch como B. elata. Irmscher (1953, p. 24) considerou B. elata uma variedade de $B$. fischeri, apesar desta variedade não ter sido validamente publicada. Por esses motivos, a exsicata Sello 135, que contêm frutos, foi selecionada como lectótipo de B. elata, e o material Luederwaldt s.n. (SP 8757), o lectótipo de Begonia fischeri var. crenatoglabra. Apesar de Golding \& Wasshausen (2002) aceitarem as variedades tovarensis, elata, brasiliensis, macroptera, ermanii, palustris, moritziana e malvacea, estabelecidas por Irmscher na chave das variedades de Begonia fischeri (1953, p. 23-24), estes táxons não foram validamente publicados, pois estão em desacordo com os Art. 33.2, 33.3 e 34.1(d) 
do Código Internacional de Nomenclatura Botânica (Greuter et al. 2003).

Begonia fischeri é uma erva com folhas transversalmente ovadas, com venação actinódroma, ala maior da cápsula com ápice agudo e sementes com ápice acuminado. É uma espécie com ampla distribução geográfica (do sul do México ao Rio Grande do Sul, no Brasil), apresentando grande variabilidade, especialmente na forma, tamanho e pilosidade das folhas. Esta elevada plasticidade induziu vários autores a estabelecerem diferentes espécies e variedades, todas correspondendo a extremos de variação (ao todo 46 nomes estão ligados a este táxon). Irmscher (1953) reconheceu 13 variedades em Begonia fischeri, baseado em características como o tamanho da folha, pilosidade e tamanho dos tricomas. A análise do material de B. fischeri não permite o reconhecimento de táxons infraespecíficos e todas as variedades propostas para esta espécie foram aqui sinonimizadas.

5. Begonia hammoniae Irmsch., Bot. Jahrb. Syst. 76(1): 54. 1953. Tipo: BRASIL. Santa Catarina: Hammonia, 1902, Luederwaldt s.n. (Holótipo SP 8783). = Begonia reitzii Brade, Sellowia 9: 31. 1958. Tipo: BRASIL. SANTA CATARINA: Itajaí, 18-II-1956, Reitz 5892 (Holótipo HBR), syn. nov.

Irmscher (1953) descreveu a placenta de Begonia hammoniae como inteira. No entanto, no holótipo, um dos lóculos tem a placenta bipartida, com lamelas diminutas. Nos demais materiais desta espécie observa-se variação no grau de união e separação das lamelas. B. hammoniae é uma espécie com elevado potencial ornamental pelas suas folhas grandes e inflorescências vistosas. O hábito da planta, a forma e o indumento da folha e a persistência das estípulas sugerem afinidades com $B$. hispida Schott, endêmica da mata atlântica do Estado do Rio de Janeiro. Entretanto, a presença de caule anguloso, folhas e estípulas menores, placenta bipartida a tripartida (característica esta muito rara nas Begonia brasileiras), e a distribuição restrita ao Estado de Santa Catarina, permite-nos considerar B. hammoniae distinta de B. hispida, em contraposição ao proposto por vários autores (Smith \& Smith 1971, Smith et al. 1986, Golding $\&$ Wasshausen 2002). Por este motivo, é proposta a reabilitação de $B$. hammoniae Irmsch., incluindo B. reitzii, anteriormente considerada sinônimo de B. hispida, em sua sinonímia.

6. Begonia hilariana A. DC., Ann. Sci. Nat., Sér. 4,
Bot. 11: 125. 1859. Tipo: BRASIL. Santa Catarina: 1816-1821, Saint-Hilaire Cat. 2. 1795 (Holótipo P; Isótipos $\mathrm{P}, \mathrm{G})$.

= Begonia raulinii Brade, Arch. Jar. Bot. Rio de Janeiro 8: 232, fig. 5, 1948. Tipo: BRASIL. SANTA Catarina: Araranguá - Maleiro, 1.II.1944, Reitz C439 (sphalm. C436) (Holótipo RB 49288; Isótipos RB 50103, HBR 723).

No Herbário do Jardim Botânico do Rio de Janeiro, não existe nenhuma coleção Reitz C436, como consta no protólogo da espécie. No entanto, foram encontradas duas exsicatas, identificadas como Reitz C439, com números de registro diferentes, um dos quais correspondendo ao do holótipo (RB 49288). Portanto, houve um erro na transcrição do número de coleta do tipo nomenclatural na obra original, que é aqui corrigido.

7. Begonia humilis Ait., Hort. Kew 3: 353. 1789. Tipo: [TRINIDAD-TOBAGO]. TRINDADE: A. Anderson s.n., introd. X-1788 by Messrs. Lee \& Kennedy" (Holótipo BM).

= Begonia alemanii Brade, Rodriguésia 18-19: 30, est. 1, fig. 1-12. 1945. Tipo: BRASIL. CEARÁ: F. Allemão \& M. de Cysneiros s.n. (Holótipo R 1352; Isótipo RB 84092), syn. nov.

Begonia humilis compartilha mesma área de distribuição geográfica e similaridades morfológicas com B. filipes Benth., da América Central, Colômbia, Venezuela e Guianas, B. prieurii A. DC., da Venezuela até a Guiana Francesa, B. semiovata Liebm., do México, norte da América do Sul, Peru e Brasil, e B. steyermarkii L.B. Sm. \& B.G. Schub., da Venezuela. Muitas destas espécies ou binômios associados a estas (p. ex., B. prieurii, B. semiovata) foram tratadas por De Candolle (1864) na seção Poecilia e caracterizadas por apresentarem placenta inteira, desigualmente bilobada a bipartida. Brade (1945) reconheceu similaridades entre B. alemanii e B. guyanensis A. DC. var. cearensis C. DC., sinonimizada sob B. humilis. Nos materiais de $B$. humilis observam-se variações no tamanho e forma dos profilos, caráter considerado por Brade no estabelecimento de B. alemanii. A análise do protólogo e dos materiais-tipo de $B$. alemanii permite-nos considerá-la sinônimo de $B$. humilis.

8. Begonia integerrima Spreng., Neue Entd. 2: 174. 1821. Lectótipo, aqui designado: BRASIL. RIO DE Janeiro: Sello s.n. (B, neg. 7529B). Síntipo remanescente: BRASIL. RIO DE JANEIRO: Sello L45O B 566 (B, neg. 7529C). 
= Begonia integerrima Spreng. var. cardioides Irmsch., Bot. Jahrb. Syst. 76(1): 71. 1953. Tipo: BRASIL. São Paulo: [Santo André], Alto da Serra [de Paranapiacaba], Edwall s.n. (Holótipo SP 8741), syn. nov.

Sprengel (1821) descreveu Begonia integerrima sem indicar o material examinado. Há no herbário de Berlim duas coleções de Sello, ambas procedentes do Rio de Janeiro. Aquela que melhor representa o protólogo de $B$. integerrima é a indicada pelo número de negativo 7529B, sendo aqui designada como lectótipo. Gomes da Silva \& Mamede (2001) reconheceram duas variedades de Begonia integerrima no Estado de São Paulo, distingüindo $B$. integerrima var. cardioides pelas folhas com base cordiforme, tépalas alvas a róseas e ramos do estilete em forma de U vs. folhas com base arredondada, tépalas vermelhas e ramos do estilete em forma de $\mathrm{V}$ na variedade típica. No entanto, esses caracteres são bastante variáveis. Estiletes em forma de $\mathrm{V}$ ou de $\mathrm{U}$ foram observados em vários materiais porém esta característica não está correlacionada a outras que justifiquem a manutenção de categorias infraespecíficas.

9. Begonia maculata Raddi, Mem. Moden. 18: 406. 1820. Gaerdtia maculata (Raddi) Klotzsch, Begoniac.: 49, est. 3A, fig. a-l. 1855. Tipo: [BRASIL. Rio dE JANEIRo] Morro do Corcovado, Raddi s.n. (Holótipo FI, n.v.), ex descr. et icon.

= Begonia aculeata Walp., Nov. Actorum Acad. Caes. Leop.-Carol. Nat. Cur. 19(1): 409. 1843 三 Gaerdtia argentea Klotzsch, Begoniac.: 50. 1855, nom. superfl. $\equiv$ Begonia maculata Raddi var. argentea (Van Houtte ex Klotzsch) A. DC. in Mart., Fl. bras. 4(1): 354. 1861. Tipo: BRASIL. Rio DE JANEIRO: XI.1830, Meyen s.n. (Holótipo B), syn. nov.

Walpers (1843) descreveu Begonia aculeata com base em material procedente do Rio de Janeiro, que posteriormente foi considerada por De Candolle (1861) como uma variedade de $B$. maculata. Este autor justificou o estabelecimento dessa variedade pela presença de pedúnculo ereto e tépalas externas ('sépalas') das flores estaminadas pouco maiores que as internas ('pétalas'). A análise do holótipo da variedade permitiu verificar que essas características, bastante variáveis, associadas ao fato desta espécie ser amplamente cultivada, não justificam a manutenção de táxons infraespecíficos, sendo portanto proposta a sinonimização da variedade.

10. Begonia platanifolia Schott in Spreng., Syst. veg. 4(2): 407. 1827. Tipo: "ex hort. Schoenbrunn, Schott s.n." (Holótipo B).

= Begonia platanifolia Otto ex Graham, Bot. Mag. 54: Tab. 3591. 1837, nom. illeg., non Schott (1827). Lectótipo, aqui designado: Graham, Bot. Mag. 54: Tab. 3591. 1837, ilustração baseada em planta coletada no Brasil, "Brasilia, hort., s.c."

Schott (1827) descreveu Begonia platanifolia sem indicar claramente o material utilizado na diagnose. Sabe-se que muitas das espécies descritas por este autor foram baseadas em material coletado por ele próprio. Há no Herbário de Berlim uma única exsicata constituída de material estéril com a indicação "ex hort. Schoenbrunn, Schott s.n.", não conflitante com o protólogo, correspondendo provavelmente ao material original utilizado na descrição da espécie.

11. Begonia radicans Vell., Fl. flumin. Icon. 10: 39. 1827 [1831]; Arq. Mus. Nac. Rio de Janeiro 5: 404. 1881. Lectótipo, aqui designado: Vellozo, Fl. flumin. Icon. 10: 39.1827 [1831], ilustração baseada em planta coletada no Brasil, Rio de Janeiro, "Pharmacopolitanis" [Parati].

= Begonia dubia Vell., Fl. flumin. Icon. 10: 42.1827 [1831]; Arq. Mus. Nac. Rio de Janeiro 5: 405. 1881, nom. illeg., non Haworth (1819). Lectótipo, aqui designado: Vellozo, Fl. flumin. Icon. 10: 42.1827 [1831], ilustração baseada em planta coletada no Brasil, Rio de Janeiro, "Pharmacopol. [Parati], juxta ostium fluvi dicti Taquary".

= Begonia procumbens Vell., Fl. flumin. Icon. 10: 36 . 1827 [1831]; Arq. Mus. Nac. Rio de Janeiro 5: 403. 1881. Lectótipo, aqui designado: Vellozo, Fl. flumin. Icon. 10: 36.1827 [1831], ilustração baseada em planta coletada no Brasil, [Rio de Janeiro], "Alpibus Fluminensibus [Serra do Mar], prope Pharmacopolitanis [Parati]".

= Begonia limmingheiana $\mathrm{E}$. Morren, La Belgique horticole 16: 21. 1866. Tipo: não indicado (Holótipo BR ?), ex descr. et icon.

= Begonia limminghei Pynaert, Rev. Hort. Belge Etrangère. 1: 259. 1875. Tipo: Limminghe s.n. (Holótipo BR ?), ex descr. et icon.

= Begonia limminghei hort ex Gentil, Pl. Cult. Serres Jard. Bot. Brux. 33. 1907. Tipo: não indicado (Holótipo $\mathrm{BR}$ ?), ex descr. et icon.

= Begonia glaucophylla Hook., Bot. Mag. 118: tab. 7219. 1892. Lectótipo, aqui designado: Hooker, Bot. Mag. 118: Tab. 7219. 1892, ilustração baseada em planta de procedência ignorada.

Begonia limmingheiana, seus sinônimos e 
variantes ortográficas, são certamente plantas mantidas apenas através de cultivo (e amplamente disseminadas), e já foram relacionadas a $B$. fagifolia Otto \& Dietr. (Pynaert 1875) e B. undulata (Hooker 1892), espécies estas fortemente rejeitadas por Weber \& Dress (1968). Uma vez que o material que originou este cultivar foi coletado no Brasil por Libon e disseminado por Jonghe de Bruxelas (Morren 1866), possivelmente a espécie selvagem seja $B$. radicans. Como o Código Internacional de Nomenclatura de Plantas Cultivadas (Trehane et al. 1995), no seu Art. 17.1, estabelece que o nome de um cultivar é composto do nome da espécie ao qual é relacionado, seguido do epíteto do cultivar, provavelmente, o nome mais correto para $B$. limmingheiana seja $B$. radicans 'Limmingheiana'. Hooker (1892) justificou o estabelecimento de uma nova espécie, Begonia glaucophylla por esta apresentar quatro tépalas ('sépalas') nas flores pistiladas, embora acreditamos que isto possa ser uma consequiência do cultivo. $\mathrm{O}$ autor não cita o tipo nomenclatural e não encontramos nenhum material no Herbário K, identificado com este nome, que pudesse corresponder ao holótipo desta espécie.

12. Begonia saxicola A. DC., Ann. Sci. Nat., Sér. 4, Bot. 11: 144, 1859. Tipo: [BRASIL. CEARÁ]: "on rocky on the Serra de Araripo [Chapada do Araripe]", 1839, Gardner 2029 (Holótipo K, foto em IPA).

= Begonia pilderifolia C. DC. in Huber, Bull. Herb. Boissier, Sér. 2, 1: 315. 1901. Tipo: BRASIL. CeArá: Serra do Baturité, IX-1897, Gardner 284 (Holótipo MG, n.v.; Isótipos $\mathrm{P}, \mathrm{G})$, syn. nov.

= Begonia heloisana Brade, Rodriguésia 18: 32, est. 5, fig. 1-9. 1945. Tipo: BRASIL. CEARÁ: Allemão 1351 (Holótipo R), syn. nov.

= Begonia burlemarxii Brade, Arch. Jar. Bot. Rio de Janeiro 15: 37, est. 6, figs. 1-11. 1957. Tipo: BRASIL. PARAíBA: cult. no Jardim Botânico do Rio de Janeiro, 1952, Burle Marx s.n. (Holótipo RB 99871), syn. nov. = Begonia egleri Brade, Arch. Jar. Bot. Rio de Janeiro 15: 38, est. 6, figs. 12-20. 1957. Tipo: BRASIL. Pernambuco: Saltinho, 2-IX-1954, Falcão 881, Egler \& Pereira (Holótipo RB), syn. nov.

Os protólogos de $B$. pilderifolia, B. heloisana, $B$. burlemarxii e $B$. egleri nos levam a considerar que estes táxons representam as mesmas variações morfológicas observadas em $B$. saxicola, principalmente no tamanho das folhas e número de tépalas das flores estaminadas e por isso estão sendo todos sinonimizados.
13. Begonia scharffii Hook., Bot. Mag. 114: 7028. 1888 $\equiv$ Begonia haageana W. Watson, Gard. Chron. III, 6(145): 388. 1889, nom. superfl. Tipo: BRASIL. SANTA CATARINA: "from the island of Desterro", 12-VIII-1887, Messrs Haage \& Schmidt [Scharff s.n.] (Holótipo K). = Begonia schenckii Irmsch., Bot. Jahrb. Syst. 76: 81. 1953. Tipo: BRASIL. SANTA CATARINA: "São Antonio auf I. Sta. Catarina”, IX-1886, Schenck 661 (Holótipo B, n.v., foto CEUL), syn. nov.

Em 1887, a empresa Haage \& Schmidt enviou duas plantas diferentes de Begonia, coletadas por Scharff, para os Jardins Botânicos de Kew e de São Petersburgo, com a solicitação de que, em se tratando de espécie(s) nova(s), recebesse $(\mathrm{m})$ o nome do coletor. Estes materiais correspondiam a táxons bastante distintos. O exemplar enviado para São Petesburgo foi descrito por Regel (1888) em novembro, como Begonia scharffiana. A planta enviada para Kew foi descrita por Hooker (1888) como B. scharffii, em dezembro daquele ano. Watson (1889) estabeleceu um novo nome para $B$. scharffii Hook., por interpretar que este nome era um homômino da espécie descrita por Regel. Apesar de parecidos (um é a forma adjetivada, e o outro, a forma substantivada do mesmo nome, Scharff), estes binômios não são homônimos e portanto, B. haageana deve ser considerado um nome supérfluo.

14. Begonia subvillosa Klotzsch, Begoniac.: 32. 1855. Tipo: BRASIL. Rio Grande do Sul: Sello d.1571 (Holótipo B).

= Begonia schmidtiana Regel, Gartenflora 28: 321, pl. 990. 1879. Tipo: BRASIL. Rio GRANdE do Sul: 10-IX-1880, Hort. Kew (Holótipo K), syn. nov.

= Begonia schmidtii Haage \& Schmidt, SamenVerzeichniss 1880: 181. 1880. Tipo: Procedência ignorada, Haage \& Schmidt s.n. (Holótipo B), syn. nov. = Begonia leptotricha C. DC., Bull. Soc. Bot. Genève Sér 2, 6: 121, pl. 4. 1914 三Begonia subvillosa Klotzsch var. leptotricha (C. DC.) L.B. Sm. \& Wassh., Phytologia 52: 446. 1983. Lectótipo, aqui designado: [PARAGUAI]. Alto Paraná, 1909-1910, Fiebrig 5707 (B; Isolectótipo B). Síntipo remanescente: [PARAGUAI]. Alto Paraná, Fiebrig 5354 (B), syn. nov.

Begonia subvillosa foi descrita por Klotzsch (1855) com base em material coletado por Sello no Rio Grande do Sul. O local de coleta deste espécime foi determinado a partir do itinerário publicado por Urban (1893). Esta espécie apresenta tricomas longos, extremamente finos, com indumento ferrugíneo variando 
de viloso ou tomentoso a glabrescente. Esta mesma variabilidade pôde ser verificada nos diferentes binômios estabelecidos por vários autores e que constam da sinonímia desta espécie.

15. Begonia ulmifolia Willd., Sp. Pl. 4: 418. 1805. Tipo: [VENEZUELA. Caracas: La Venta] "In America meridionali", Humboldt 690 (Holótipo B, Cat. 17571; Isótipos B, E, P n.v.).

= Begonia dasycarpa A. DC., Ann. Sci. Nat., Bot. Sér. 4, 11: 127. 1859. Tipo: BRASIL. BAHIA: 1851, Dupasquier s.n. (Holótipo G-DC), syn. nov.

= Begonia jairii Brade, Arch. Jar. Bot. Rio de Janeiro 10: 135, pl. 5. 1950. Tipo: BRASIL. Espírito SANTo: Estrada de Ferro Vitória-Minas Gerais, entre Boa Vista e Estação Pedro Palácio, Vieira 44 \& Mendonça (Holótipo RB; Isótipo HB), syn. nov.

Begonia ulmifolia apresenta distribuição disjunta, ocorrendo no Norte da América do Sul e Leste do Brasil. Possui caule anguloso, notadamente nas partes adultas, folhas obovado-oblongas, com base assimétrica e pubescentes em ambas as faces. A análise dos protólogos de $B$. dasycarpa e $B$. jairii não deixa dúvidas de que correspondem à mesma espécie, sendo, portanto, aqui sinonimizadas.

16. Begonia undulata Schott in Spreng., Syst. veg. 4(2): 408, 1827 [Janeiro] झ Gaerdtia undulata (Schott) Klotzsch, Begoniac.: 50. 1855. Tipo: BRASIL. Schott s.n. ex hort. Schönbrunn (Holótipo B).

= Begonia undulata Otto ex Graham, Bot. Mag. 54: tab. 2723. 1827, nom. illeg., non Schott (1827) $\equiv$ Trilomisa undulata (Graham) Raf., Flora Telluriana. 2: 91. 1836. Tipo: BRASIL. Graham s.n. (Holótipo K). = Begonia salicifolia A. DC., Ann. Sci. Nat., Bot. Sér. 4, 11: 128. 1859. Tipo: BRASIL. [RIo DE JANEIRo]: Macaé, Riedel 99 (Holótipo LE, n.v.; Isótipo G-DC), syn. nov.

Rafinesque (1836) considerou Begonia undulata Otto ex Graham pertencente ao gênero Trilomisa, descrito naquela obra. Ao indicar o tipo desta combinação nova, fez referência direta à prancha 2723 (Graham 1827). De Candolle (1859) descreveu Begonia salicifolia a partir de material procedente do Rio de Janeiro. Anos mais tarde, este autor (De Candolle 1861, p. 355) considerou esta espécie semelhante a B. undulata, exceto pelas folhas estreitamente alongadas. A análise do holótipo evidencia o extremo da variação do tamanho e largura das folhas desta espécie. Desta forma, é proposta a sinonimização de B. salicifolia sob B. undulata.
Táxons pouco conhecidos ou de identidade duvidosa

1. Begonia corallina Carrière, Rev. Hort. 47: 89. 1875. Tipo: Procedência ignorada, s.c. (Holótipo P, não localizado).

Carrière (1875) descreveu Begonia corallina, citando o Brasil como a provável procedência desta espécie. Barkley (1972) posicionou-a na Seção Gaerdtia, que agrupa apenas espécies brasileiras. Smith et al. (1986) consideraram B. corallina sinônimo de B. maculata. Os poucos materiais desta espécie, depositados nos herbários visitados e cultivados no Jardim Botânico de Kew, foram por nós identificados como B. maculata. A análise do protólogo desta espécie não permite associá-la com segurança a $B$. maculata, e portanto $B$. corallina foi considerado um nome de aplicação duvidosa.

2. Begonia cucullifolia Hassk., Hort. Bogor. Descr.: 311. 1858. Tipo: "Cult. hort. bot" (Holótipo não localizado).

Hasskarl (1858) referiu este binômio sob B. cucullata Willd. Uma vez que não localizamos o holótipo, preferiu-se retirar este binômio da sinonímia de B. cucullata, pois em nossa opinião é necessário examinar a semente para que sua identidade seja reconhecida.

3. Begonia cuneata Walp., Repert. bot. syst. 2: 214. 1843, pro syn. Begonia spatulata Willd. ex Spreng. 1825.

Este binômio foi tratado por Smith et al. (1986) como sinônimo de Begonia cucullata Willd. var. spatulata (Loddiges) Golding. No entanto, a não localização da obra princeps, impede-nos de tomar uma decisão se este binômio deveria ou não estar associado a B. cucullata Willd.

4. Begonia dichroa Sprague, Bull. Misc. Inform. 251. 1908. Tipo: BRASIL. Haage \& Schmidt Erfurt (Holótipo $\mathrm{K}$, não localizado).

Sprague (1908) descreveu Begonia dichroa distingüindo-a de $B$. maculata pela disposição dos óvulos em ambas as faces das lamelas (vs. apenas na face externa) e pelas folhas menos oblíquas (vs. fortemente oblíquas). Na ilustração desta espécie apresentada em Smith et al. (1986), verificou-se que o hipanto (orbicular) é diferente do de B. maculata (oboval). Considerando que este nome não foi referido em nenhum trabalho 
posterior à sua publicação original e que nos herbários visitados, não foi encontrado nenhum material identificado com este nome ou que pudesse corresponder ao material-tipo desta espécie, preferiu-se considerar B. dichroa como um nome de aplicação duvidosa.

5. Begonia dispar Rchb., Mith. Landw. 54. 1829, fide Smith et al. (1986).

Este binômio é citado em Smith et al. (1986) como sinônimo de B. cucullata Willd. var. cucullata, apesar dos autores não terem consultado a obra princeps. No levantamento bibliográfico realizado, não foi localizada nenhuma obra de Reichenbach com as referências especificadas. B. dispar está sendo considerada neste trabalho como um táxon de identidade duvidosa.

6. Begonia meyeniana Walp., Nov. Actorum Acad. Caes. Leop.-Carol. Nat. Cur. suppl. 2 (19, suppl. 1): 409. 1843. Tipo: BRASIL. RIO DE JANEIRO: Meyen s.n. (Síntipo B, não localizado); Guiana Anglica [GUIANA], Schomburg s.n. (Síntipo B, não localizado).

Um dos síntipos de Begonia meyeniana (Meyen s.n.) foi coletado no Rio de Janeiro. Nos comentários desta espécie, De Candolle (1864, p. 395) considerou $B$. meyeniana um táxon duvidoso, reconhecendo similaridades com $B$. humilis, mas não o considerou como sinônimo desta, por duvidar que a distribuição geográfica desta última atingisse o Rio de Janeiro. Somente a localização e análise dos materiais-tipo de B. meyeniana poderão esclarecer a verdadeira identidade deste táxon.

7. Begonia patula Haw., Suppl. pl. succ.: 100. 1819. Tipo: “Cult. apud Loddiges' Nursery A.D.1816, introd. a Dom Guilermo Anderson, a Dom Otto, ex Horto Regio Berolinense, A.D. 1811" (Holótipo OXF ?).

Segundo Stafleu \& Cowan (1979), o herbário de Haworth foi comprado pelo herbário Fielding (OXF) o qual manteve parte de sua coleção para estudo, descartando a maioria dos espécimes. Alguns espécimes de Aloe, Gasteria e Haworthia, etiquetados por Haworth, são encontrados ainda no herbário Fielding. Haworth mantinha um jardim em Chelsea, Inglaterra, onde cultivava plantas suculentas (1817-1833). Pode-se presumir que, como era comum entre botânicos que trabalhavam com plantas suculentas, Harworth nem sempre fazia exsicatas das espécies novas que cultivava. De acordo com esses autores, Desmond (apud Stafleu \& Cowan 1979) informa que plantas de
Haworth podem ser encontradas em K, porém nenhuma exsicata com identificação positiva desta espécie foi localizada naquele herbário. Begonia patula era um nome conhecido desde 1811 , através de materiais cultivados e disseminados em vários jardins botânicos europeus, tendo sido efetivamente publicado apenas em maio de 1819 (Stearn 1938, Golding 1981). Este binômio tem sido referido em literatura muito esporadicamente. Klotzsch (1855, p. 30) tratou um material coletado no Brasil por Sello como B. patula Haw., mas, posteriormente, seu conceito desta espécie foi sinonimizado sob B. fischeri. Irmscher (1953, p. 12) considerou B. patula como sinônimo de $B$. fischeri, por considerar este último o nome mais antigo. Smith \& Smith (1971, p. 49) aceitaram B. patula, incluindo $B$. fischeri como sinônimo desta. Posteriormente, reconheceram B. fischeri, e incluíram $B$ patula Haw. na sinonímia de $B$. populifolia sensu Liebm. (Smith et al. 1986) e de B. fischeri var. fischeri (Golding \& Wasshausen 2002), trazendo mais confusão ao problema. Begonia patula Haw. foi publicada com uma diagnose incompleta $[B$ (spreading) foliis inaequalitis cordatis, $s$. fere semicordatis crenoetis fere obtusiusculis perviridibus junioribus utrinque parce villosulis petiolis vilosis], e ausência de ilustração e de citação de material-tipo. A aplicação deste epíteto em $B$. fischeri tem gerado dúvidas quanto à identidade destas espécies. Se o holótipo de B. patula for localizado e comprovar tratarem-se de um mesmo táxon, será proposta por nós a rejeição deste em favor de $B$. fischeri, um nome mais amplamente utilizado em literatura e disseminado nos diversos herbários. Com base nas informações disponíveis até o momento, B. patula Haw. é aqui considerado um nome de aplicação duvidosa.

8. Begonia sandersii Hort. Kew ex A. DC., Prodr. 15(1): 400. 1864, nom. nud.

Irmscher (1953) foi o primeiro a considerar Begonia sandersii sinônimo de Begonia limmingheiana E. Morren. Comentou nesta obra que fora encontrada no Herbário de Berlim uma exsicata estéril desta espécie que continha a observação "Vinda de DC como um híbrido cujos parentais são desconhecidos, h. B. 1874". Comentou ainda que a etiqueta fora descrita por Alexander Braun e que o material proviera do herbário deste (Irmscher 1953, p. 29). Smith et al. (1986) atribuíram a autoria de Begonia sandersii a A. De Candolle, tratando-a como um sinônimo de B. radicans Vell. De Candolle (1864) 
nunca descreveu tal espécie, apenas citou-a como uma espécie duvidosa. No herbário G-DC, há uma exsicata, identificada como B. sandersii, analisada por De Candolle em cuja etiqueta está grafado "Hort. Kew, 1837", a qual é composta de um ramo frutífero com três folhas. A forma das folhas e das alas dos frutos é bastante distinta de $B$. radicans e das espécies relacionadas a esta (B. solananthera A. DC. e $B$. integerrima Spreng.). Por este motivo, retirou-se Begonia sandersii da sinonímia de $B$. radicans.

Táxons excluídos

1. Begonia albopicta W. Bull., Cat. New, Beautiful \& Rare Pl. 210: 13. 1885. Begonia maculata Raddi var. albopicta (W. Bull.) Fotsch, Die Begonien: 93, Abb. 40. 1933. Tipo: não indicado.

Em 1885, William Bull publicou Begonia albopicta num catálogo de plantas cultivadas, citando-a como uma espécie brasileira (fide Irmscher 1960, p. 79). No ano seguinte, Regel (1886) comentou que B. albopicta poderia ser considerada uma forma de B. maculata, com folhas menores, entretanto preferiu mantê-la na categoria de espécie. Fotsch (1933) considerou B. albopicta uma variedade de B. maculata diferenciando-a das outras variedades pelas folhas menores, com manchas estreitas, e apresentou uma foto de uma planta estéril e cultivada (foto 40) para a ilustração da variedade. De acordo com Irmscher (1960, p. 79), esta ilustração não corresponde nem a B. maculata nem a $B$. albopicta, mas sim a um híbrido entre B. albopicta e B. olbia Kerchove (B. × argenteoguttata hort.) (Smith et al. 1986).

2. Begonia coccinea 'Comte Alfred de Limering' in Regel, Gartenflora 17: 194, pl. 584. 1868. Tipo: não indicado.

3. Begonia coccinea 'A. de Liming' in Regel, Gartenflora 17: 194, fig. 584. 1868. Tipo: não indicado.

4. Begonia glaucophylla 'Scandens' in Hort P. Henderson, Spring Catalogue of New, Rare and Beautiful Plants 1872: 1. 1872. Tipo: não indicado.

5. Begonia glaucophylla 'Splendens' in W. Robinson, The Garden 19: 166. 1881. Tipo: não indicado.

Excluídas da sinonímia de B. radicans Vell. por tratarem-se de cultivares.

6. Begonia glaucophylla 'Scandens' in Fotsch, Die Begonien: 26. 1933. Tipo: não indicado.
7. Begonia glaucophylla 'Splendens' in Fotsch, Die Begonien: 26: 1933. Tipo: não indicado.

Excluídas da sinonímia de Begonia glaucophylla Hook. (= B. radicans) por tratarem-se de cultivares.

8. Begonia maculata Raddi var. elegantissima Fotsch, Die Begonien: 93. 1933. Tipo: não indicado.

9. Begonia maculata Raddi var. wrightii Fotsch, Die Begonien: 93. 1933. Tipo: não indicado.

Fotsch (1933) descreveu estas variedades a partir de material cultivado. Nos herbários visitados (B, G, K, $\mathrm{P})$, não foram encontrados materiais de Begonia maculata identificados como estas variedades. Desta forma, estes táxons estão sendo considerados prováveis cultivares.

Agradecimentos - As autoras agradecem aos curadores dos herbários indicados no texto por disponibilizarem suas coleções. Ao Instituto de Botânica pela utilização de suas instalações físicas. Ao Dr. Jefferson Prado e aos assessores pelas valiosas sugestões e críticas ao manuscrito. À Capes e à Fundação Botânica Margaret Mee pela bolsa PICDT e auxílio concedidos à primeira autora e ao $\mathrm{CNPq}$ pela bolsa de produtividade em pesquisa concedida à segunda autora.

\section{Referências bibliográficas}

BARANOV, A. \& BARKLEY, F.A. 1974. The sections of the genus Begonia. Northeastern University. Boston.

BARKLEY, F.A. 1972. Key to the sections of the Begoniaceae. The Buptonian 1:1-7.

BRADE, A.C. 1945. Begônias novas do Brasil, IV. Rodriguésia 18-19:23-33, fig. 1-6.

BRADE, A.C. 1948. Begônias novas do Brasil, V. Arquivos do Jardim Botânico do Rio de Janeiro 8:227-247.

CARAUTA, J.P.P. 1973. The text of Vellozo's Flora Fluminensis and its effective date of publication. Taxon 22:281-284.

CARRIÈRE, É.-A. 1875. Begonia corallina. Revue Horticole 47:89.

DE CANDOLLE, A. 1859. Mémoire sur la famille du Begoniacées. Annales des Sciences Naturelles Botanique, Sér. 4, 11:93-149.

DE CANDOLLE, A. 1861. Begoniaceae. In Flora brasiliensis (C.P.F. Martius, ed.). F. Fleischer, Lipsiae, v.4, pars 1, p. 338-396.

DE CANDOLLE, A. 1864. Begoniaceae. In Prodromus Systematis Naturalis Regni Vegetabilis (A. De Candolle, ed.). Victoris Masson et Fils, Paris, v.15, pars 1, p.266-408.

DE LANGE, A. \& BOUMAN, F. 1999. Seed micromorphology of Neotropical Begonias. Smithisonian Contributions to Botany 90:1-49. 
DOORENBOS, J., SOSEF, M.S.M. \& DE WILDE, J.J.F.E. 1998. The sections of Begonia. Wageningen Agricultural University Papers 98:1-266.

FOTSCH, K.A. 1933. Die Begonien: ihre Beschreibung, Kultur, Züchtung und Geschichte. Verlag von Eugen Ulmer, Stuttgart, p. 91-93.

GOLDING, J. 1981. How B. fischeri got its right name. Begonian 49:7.

GOLDING, J. \& WASSHAUSEN, D.C. 2002. Begoniaceae, Edition 2. Part I: Annotated Species List. Part II: Illustrated Key, Abridgement and Supplement. Smithsonian Contributions from the United States National Herbarium 43:1-289.

GOMES-DA-SILVA, S.J. \& MAMEDE, M.C.H. 2001. Begoniaceae da Mata Atlântica na Serra do Mar do Estado de São Paulo, Brasil. Boletim do Instituto de Botânica 15:1-61.

GRAHAM, R.C. 1827. Begonia undulata. Botanical Magazine 54: tab. 2723.

GREUTER, W., MCNEILL, J., BARRIE, F.R., BURDET, H.M., DEMOULIN, V., FILGUEIRAS, T.S., NICOLSON, D.H., SILVA, P.C., SKOG J.E., TREHANE, P., TURLAND, N.J. \& HAWKSWORTH, D.L. (eds.). 2003. Código Internacional de Nomenclatura Botânica (Código de Saint Louis, 2000) adotado pelo XVI Congresso Internacional de Botânica, Saint Louis, Missouri, julhoagosto de 1999. Tradução de C.E.M. Bicudo \& J. Prado. Instituto de Botânica, International Association for Plant Taxonomy e Sociedade Botânica de São Paulo, São Paulo.

HASSKARL, J.C. 1858. Begonia cucullifolia. Hortus Bogoriensis Descriptus:311.

HOOKER, J.D. 1888. Begonia scharfii. Botanical Magazine 114: tab. 7028.

HOOKER, J.D. 1892. Begonia glaucophylla. Botanical Magazine 118: t. 7219.

IRMSCHER, E. 1953. Systematische studien über Begoniaceen des tropischen Sudamerikas besonders Brasiliens. Botanische Jarbücher für Systematic Pflanzengeschichte und Pflanzengeographie 76:1-102.

IRMSCHER, E. 1960. Fam. Begoniaceae, Schiefblattgewächse. Pareys Blumengärt 2a. ed., p. 67-91.

JACQUES, E.L. 2002. Estudos taxonômicos das espécies brasileiras do gênero Begonia L. (Begoniaceae) com placenta partida. Tese de doutorado, Universidade de São Paulo, São Paulo.

JACQUES, E.L. \& MAMEDE, M.C.H. 2004. Novelties in Begonia (Begoniaceae) from the coastal forests of Brazil. Brittonia 56:75-81.
KLOTZSCH, J.F. 1855. Begoniaceen - Gattungen und Arten. Nicolaischen Buchhandlungen, Berlin.

MORREN, E. 1866. Le Begonia Comte Alfred de Limminghe. La Belgique Horticole 16:21-22.

PYNAERT, E. 1875. Le Begonia Limminghi. Revue Horticulture Belge et Étrangère 1:259-260.

RAFINESQUE, C. 1836. Trilomisa undulata. Flora Telluriana 2a part:91.

REGEL, E. 1886. Neue und empfehlenswerthe Pflanzen. Gartenflora 35:402.

REGEL, E. 1888. Neue und empfehlenswerthe Pflanzen. Gartenflora 37:127.

SCHOTT, H. 1827. Fasciculus plantarum brasiliensium. Systema Vegetabilium 4:403-410.

SMITH, L.B. \& SMITH, R.C. 1971. Begoniáceas. In Flora Ilustrada Catarinense (R. Reitz, ed.). Herbário Barbosa Rodrigues, Itajaí, fasc. BEGO p.1-128.

SMITH, L.B., WASSHAUSEN, D.C. GOLDING, J. \& KAREGEANNES, C.E. 1986. Begoniaceae: Part. I: Illustrated Key. Part II: Annotated Species List. Smithsonian Contributions to Botany 60:1-584.

SPRAGUE, T.A. 1908. Begonia dichroa. Bulletin of Miscellaneous Information 6:251.

SPRENGEL, K. 1821. Neue Entdeckungen im ganzen Umfang der Pflanzenkunde. Friedrich Fleischer, Leipzig. v.2.

STAFLEU, R.A. \& COWAN, R.S. 1979. Taxonomic Literature. $2^{\mathrm{a}}$ ed. Bohn, Scheltema \& Holkema ed., Utrecht, v.2.

STEARN, W.T. 1938. Bibliographical Notes: CVII. Haworth's Supplementum Plantarum Succulentarum. London Journal of Botany 76:114.

TAUBERT, P. 1896. Begoniaceae. Botanische Jarbücher für Systematic Pflanzengeschichte und Pflanzengeographie 21:445.

TREHANE, P., BRICKELL, C.D., BAUM, B.R., HETTERSCHEID, W.L.A., LESLIE, A.C., McNEILL, J., SPONGBERG, S.A. \& VRUGTMAN, F. (eds.). 1995. International Code of Nomenclature for Cultivated Plants. Quaterjack Publ., Winborne.

URBAN, I. 1893. Biographische Skizzen. Botanische Jarbücher für Systematic Pflanzengeschichte und Pflanzengeographie 17:177-198.

WALPERS, W.G. 1843. Begoniaceae. Novorum Actorum Academiae Caesareae Leopoldino-Carolinae Naturae Curiosorum. 19, Supl. 1:408-409.

WATSON, W. 1889. Begonia scharffiana. The Gardeners Chronicle 6:388.

WEBER, C. \& DRESS, W.J. 1968. Notes on the nomenclature of some cultivated Begonias (Begoniaceae). Baileya $16: 42-73$. 